\title{
Use of Flexible Body Coupled Loads in Assessment of Day of Launch Flight Loads
}

\author{
Brett R. Starr ${ }^{1}$ and Isam Yunis ${ }^{2}$ \\ NASA Langley Research Center, Hampton, VA 23681 \\ Aaron D. Olds ${ }^{3}$ \\ Analytical Mechanics Associates, Hampton, VA 23681
}

\begin{abstract}
A Day of Launch flight loads assessment technique that determines running loads calculated from flexible body coupled loads was developed for the Ares I-X Flight Test Vehicle. The technique was developed to quantify DOL flight loads in terms of structural load components rather than the typically used q-alpha metric to provide more insight into the DOL loads. In this technique, running loads in the primary structure are determined from the combination of quasi-static aerodynamic loads and dynamic loads. The aerodynamic loads are calculated as a function of time using trajectory parameters passed from the DOL trajectory simulation and are combined with precalculated dynamic loads using a load combination equation. The potential change in aerodynamic load due to wind variability during the countdown is included in the load combination. In the event of a load limit exceedance, the technique allows the identification of what load component is exceeded, a quantification of how much the load limit is exceeded, and where on the vehicle the exceedance occurrs. This technique was used to clear the Ares I-X FTV for launch on October 28, 2009. This paper describes the use of coupled loads in the Ares I-X flight loads assessment and summarizes the Ares I-X load assessment results.
\end{abstract}

\section{Nomenclature}

CLA $=$ Coupled Loads Analysis

C.G. $\quad=$ Center of Gravity

$\mathrm{CM} \quad=$ Crew Module

$\mathrm{CR} \quad=$ Critical Ratio

DOF $\quad=$ Degrees of Freedom

DOL $=$ Day of Launch

FS $\quad=$ First Stage

FTV $=$ Flight Test Vehicle

$F_{X} \quad=X$ component of the coupled load forces

$F_{Y} \quad=\quad Y$ component of the coupled load forces

$F_{Z} \quad=\quad Z$ component of the coupled load forces

GRAM $=$ Global Reference Atmospheric Model

$i \quad=$ coupled load index

IPT $=$ Integrated Product Team

JSC $=$ Johnson Space Center

KSC $=$ Kennedy Space Center

LAS $=$ Launch Abort System

LRFE $=$ Low Resolution Flight Element

MSFC $=$ Marshall Space Flight Center

$M_{X} \quad=$ Coupled load Moment about the FTV X axis

${ }_{2}^{1}$ Aerospace Engineer, Vehicle Analysis Branch, 1 North Dryden Road, Mail Stop 451, Member

${ }^{2}$ Aerospace Engineer, Structures and Thermal Systems Branch, 1 North Dryden Road, Mail Stop 431, Senior Member

${ }^{3}$ Aerospace Engineer, 1 North Dryden Road, Mail Stop 451

American Institute of Aeronautics and Astronautics 


$\begin{array}{ll}M_{Y} & =\text { Coupled load Moment about the FTV Y axis } \\ M_{Z} & =\text { Coupled load Moment about the FTV Z axis } \\ N_{S} & =\text { Shear running load } \\ N_{X} & =\text { Axial running load } \\ \text { PMBT } & =\text { Propellant Mean Bulk Temperature } \\ \text { POST } & =\text { Program to Optimize Simulated Trajectories } \\ \text { RoCS } & =\text { Roll Control System } \\ \text { RSRM } & =\text { Reusable Solid Rocket Motor } \\ \text { RSS } & =\text { Root Sum Square } \\ \text { SSP } & =\text { Space Shuttle Program } \\ \text { STEL } & =\text { Static Elastic } \\ \text { t } & =\text { time } \\ \text { TO } & =\text { Thrust Oscillation } \\ \text { US } & =\text { Upper Stage } \\ \text { USS } & =\text { Upper Stage Simulator } \\ \text { VAB } & =\text { Vehicle Assembly Building }\end{array}$

\section{Introduction} res I-X was a full scale flight test of the Ares I Crew Launch Vehicle designed to transport crew to low Earth
morbit. Ares I was designed as a two-stage-to-orbit vehicle that utilized a five-segment reusable solid rocket
motor (RSRM) First Stage (FS) derived from the Space Shuttle Program (SSP) four-segment RSRM and a liquid
propellant Upper Stage (US) with a J2-X engine derived from the J2 engine flown on the Saturn V. The Ares I-X
Flight Test Vehicle (FTV) was geometrically similar to Ares I but used different propulsion because the
development of the Ares I propulsion elements was not completed at the time of the test flight. The Ares I-X FS
used an existing SSP four-segment RSRM with an inert fifth segment to maintain geometric similarity with the Ares
I five-segment FS and its US was an inert Upper Stage Simulator (USS) of the Ares I US with no primary stage
propulsion. The FTV is shown in Fig 1. The test flight launched successfully from Kennedy Space Center (KSC)
Launch Complex 39, Pad B on October 28, 2009.

A primary objective of the test flight was to demonstrate controllability of the Ares I design during its boost phase. Demonstrating controllability was important because the long, slender geometry resulted in an aerodynamically unstable and flexible vehicle with structural modes that could be excited by its thrust vector control system. A robust flight control system was developed to maintain a prescribed vehicle attitude as a function of altitude without large overshoots or oscillations, while damping the vehicle's first bending mode to prevent adverse interactions between its flexible structure and thrust vector control and wind gusts.

In order to demonstrate controllability, the aerodynamic loading needed to be similar to that of the operational vehicle. The Ares I-X trajectory was developed to provide similar aerodynamic loads by matching the Ares I dynamic pressure versus Mach number (Mach-q) relationship to within 10\% through Mach 4. The four-segment RSRM did not have sufficient impulse to produce an Ares I similar Mach-q relationship beyond Mach 4. The RSRM reached burnout near Mach 4.6 and separation occurred at approximately $129 \mathrm{kft}$, well below the Ares I Mach 6.1, $184 \mathrm{kft}$ separation point. After separation, both the FS and USS descended uncontrolled to water impact. A plot of the trajectory is shown in Fig. 2. A summary of the trajectory events is given in Table 1. The FTV trajectory development is discussed in detail in Ref. 1.

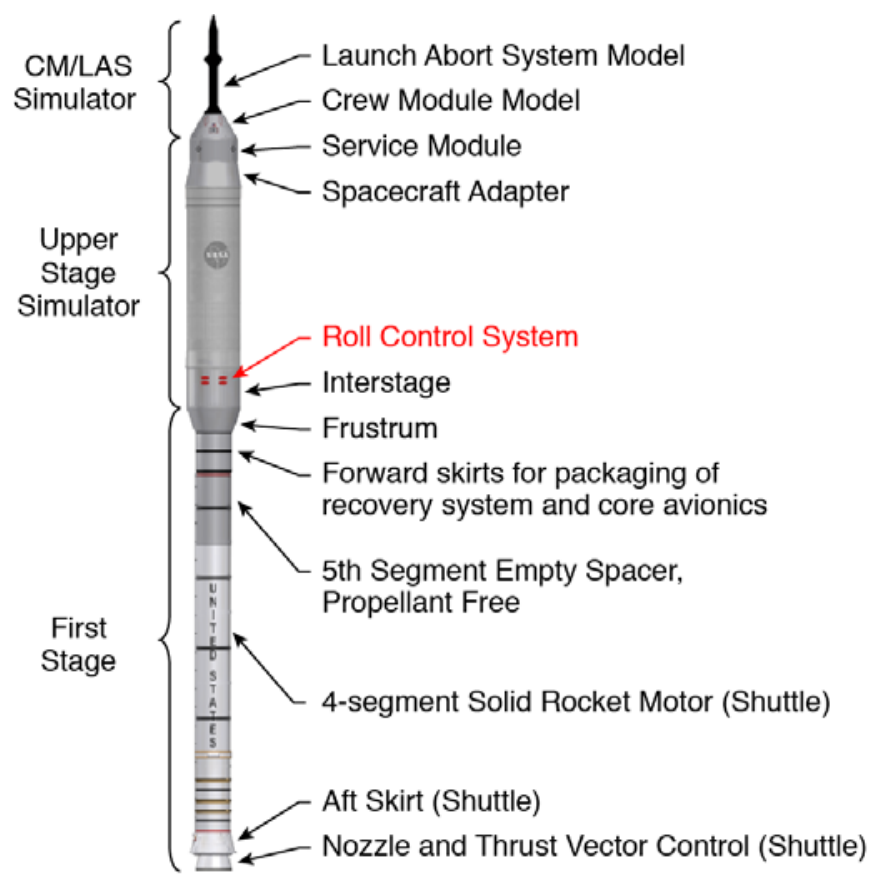

Figure 1. Ares I-X Flight Test Vehicle 


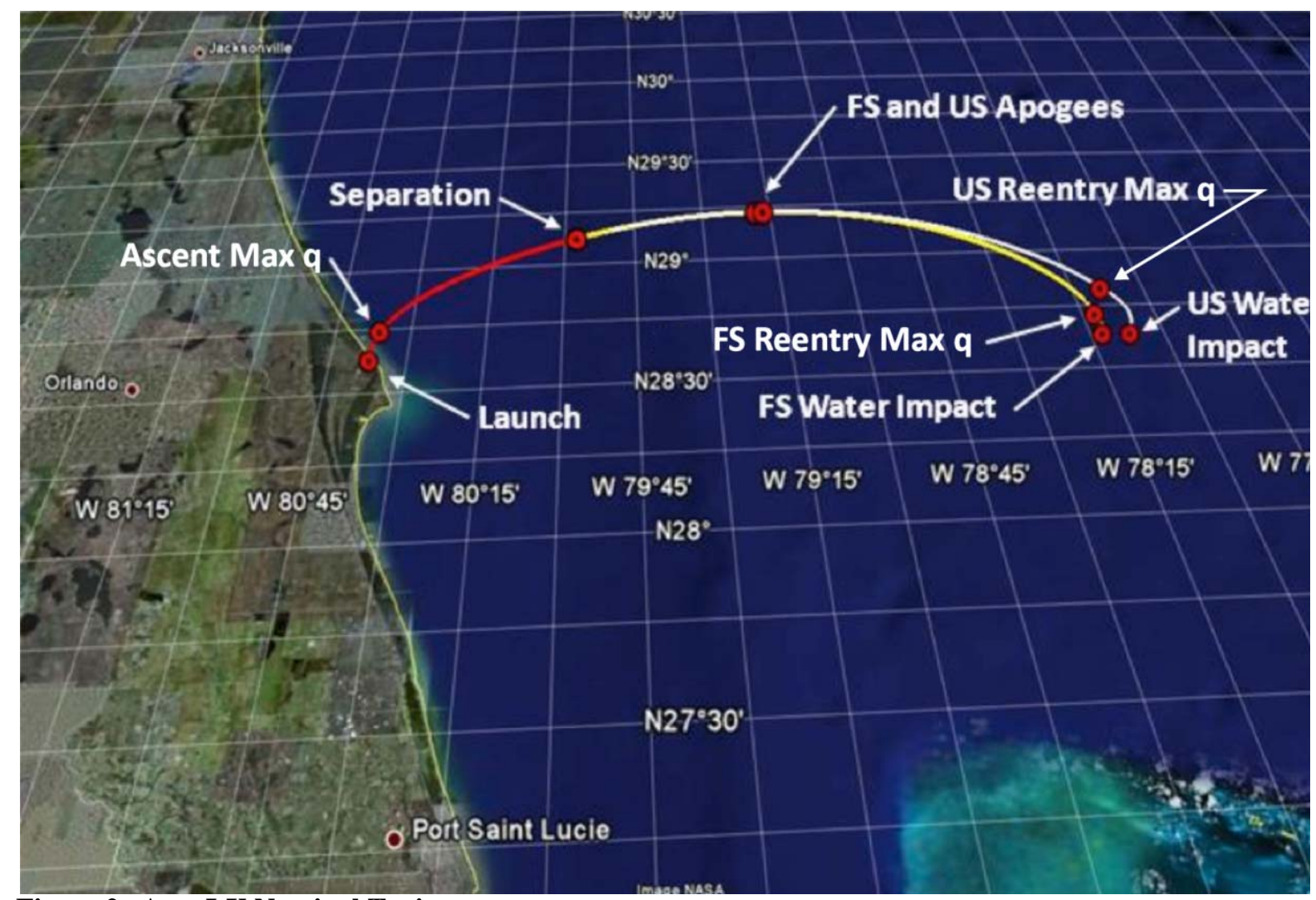

Figure 2. Ares I-X Nominal Trajectory

Table 1 Summary of Ares I-X Test Flight Trajectory Events

\begin{tabular}{|l|c|c|c|c|}
\hline Event & Time (s) & Down Range (nmi) & Altitude (kft) & q (psf) \\
\hline Ascent max q & 58 & 3.8 & 38.9 & 874 \\
\hline Separation & 123 & 36.4 & 128.6 & 102 \\
\hline USS apogee & 159 & 63.9 & 148.9 & 37 \\
\hline FS apogee & 160 & 63.9 & 149.1 & 35 \\
\hline USS reentry max q & 249 & 122.6 & 42.7 & 2355 \\
\hline FS reentry max q & 254 & 119.1 & 51.8 & 1060 \\
\hline USS water impact & 310 & 130.8 & 0 & 503 \\
\hline FS water impact & 352 & 122.1 & 0 & 13 \\
\hline
\end{tabular}

The steering commands required to match the Ares I Mach-q were developed for monthly mean wind and atmospheric properties. Monthly steering tables were developed for launches from July to November, 2009. The steering commands were not updated prior to launch based on knowledge of DOL conditions. Instead, the FTV was designed to accommodate monthly three-sigma wind and atmospheric dispersions. This approach required a DOL assessment to verify the primary structure's load capacity would not be exceeded at any point in the ascent due to DOL winds aloft and atmosphere. 


\section{DOL Flight Load Assessment}

A DOL loads assessment was developed that quantified loads in the primary structure in terms of running loads calculated from coupled loads. Coupled loads are forces and moments resulting from the combination of quasi-static aerodynamic loads acting on the FTV and dynamic loads, some of which excite the FTV's natural frequencies. Running loads are the combination of forces and moments expressed as force per unit width of the structure, $\mathrm{lb}_{\mathrm{f}} / \mathrm{in}_{\mathrm{in}}$. Normal stress running loads combine axial force and bending moments. Shear stress running loads combine transverse shear and torsion. Running loads are used by design engineers to define the load carrying capacity of structures.

The assessment determined running loads from the combination of aeroelastic loads that resulted from quasistatic aerodynamic loads acting on the flexible FTV and dynamic loads due to wind gusts, aeroacoustic buffeting, roll control system (RoCS) firings, thrust vectoring during maneuvering, and RSRM internal acoustic modes. Aeroelastic loads were determined using a static-elastic (STEL) analysis and are referred to as STEL loads. STEL loads were a function of trajectory parameters such as dynamic pressure, total angle of attack, and Mach number, and hence, were a function of DOL winds and atmospheric conditions (density, pressure, temperature, speed of sound). The DOL STEL loads were computed using inputs from a 6-DOF trajectory simulation updated with DOL winds and atmospheric conditions measured with balloons released prior to launch. The dynamic loads were not affected by the DOL conditions and were calculated pre-flight. The coupled loads (forces and moments) were determined by combining the DOL STEL loads with the dynamic loads using a loads combination equation. Axial compressive, axial tensile, and shear running loads were computed from the coupled loads and compared to the primary structure's load limits in terms of a critical ratio (CR), where the ratio was defined as the DOL running load component divided by the structure's component load limit. The launch commit criterion for flight loads was defined as a CR of 1.0 or less for all load components. The loads assessment process is summarized in the following steps. Each step is discussed in later sections.

1. Measure DOL winds and atmospheric conditions and develop winds and atmospheric models.

2. Perform an ascent trajectory simulation to define flight parameters necessary to compute STEL loads.

3. Perform a STEL analysis to determine DOL STEL loads.

4. Compute the coupled loads from the combination of DOL STEL and pre-calculated dynamic loads.

5. Compute running loads from the coupled loads forces and moments

6. Compute CRs from the running load components and FTV load limits

7. Determine the trajectory go/no-go decision based on the CR launch commit criteria.

The FTV assessment determined running loads rather than using a q-alpha load indicator as a means of providing more insight into DOL loads. The running loads quantified the load state along the entire length of the FTV and were directly comparable to the structure's load limits. In the event of an exceedance, the location of the exceedance, the type of load limit exceeded, and the magnitude of the exceedance as a percentage of the structure's strength could be determined from the running loads. Use of a q-alpha load indicator cannot provide any information regarding an exceedance's location, load type, or magnitude.

The FTV running load limits were based on design requirements determined in pre-flight analyses. Integrated Product Teams (IPT) designed and fabricated the structure to meet the requirements. Because the load limit varied as a function of position on the FTV, it was not feasible to design the structure such that its actual strength matched the load requirement at each point. At numerous locations, the actual strength exceeded the limit.

In cases where the exceedance was known to be small relative to the structure's load limit, a waiver to the loads launch commit criteria could be requested. The running load information was beneficial for developing rationale for a waiver to loads launch commit criteria. The IPT responsible for certifying the FTV's load capacity to meet the exceedance could compare the DOL running load against the structure's actual load limit and make an informed decision on whether or not to allow the exceedance. If approved, the launch could proceed and thereby save launch cost.

\section{A. DOL Winds}

1. Wind and Atmosphere Measurement

The Natural Environments Branch at Marshall Space Flight Center (MSFC) working in conjunction with the Ares I-X Systems Engineering and Integration team developed an upper atmospheric monitoring process to support the DOL flight loads assessment ${ }^{2}$. Winds aloft and atmospheric thermodynamic properties were measured during the launch countdown using Automated Meteorological Profiling System low resolution flight elements (LRFE). The LRFE consisted of a weather balloon with a radiosonde that measured the atmospheric conditions and tracked the balloon's position with a Global Positioning System tracking device. The LRFE had a maximum altitude of 
approximately $100 \mathrm{kft}$ and could reach that altitude within approximately 90 minutes. In the event of a launch delay, LRFE releases would continue to support assessments up to the close of that day's launch window.

The balloon release schedule developed for DOL evaluations is shown in Fig. 3. Each release consisted of a primary balloon and a backup balloon to mitigate the risk from balloon failure. Backup balloons were released 5 minutes after the primary and are denoted with an ' $\alpha$ ' in Fig. 3. The first release was at eight hours before launch (L8:00) to allow time for the LRFE to reach its maximum altitude and the completion of an initial assessment prior to starting the launch countdown. The initial load assessment coupled with a forecast of winds aloft was used to provide a recommendation on whether or not to start the launch countdown. If a flight load was at or above the FTV load limit and the forecast did not indicate an improvement in winds, it was recommended that the launch countdown not be started. Once the countdown started, balloons were released every hour. Hourly releases provided sufficient time between balloons to complete a load assessment and allowed for completion of all countdown assessments within three hours of launch. However, the altitude reached by the LRFE at the time of countdown assessments was reduced to $65 \mathrm{kft}$. The LRFEs continued to rise during the assessments and made measurements up to their maximum altitude. Load assessments were completed within three hours of flight because the effect of wind change over time on STEL loads was determined for a period of 3.5 hours. This allowed for the launch to proceed if any one of the load assessments was successfully completed.

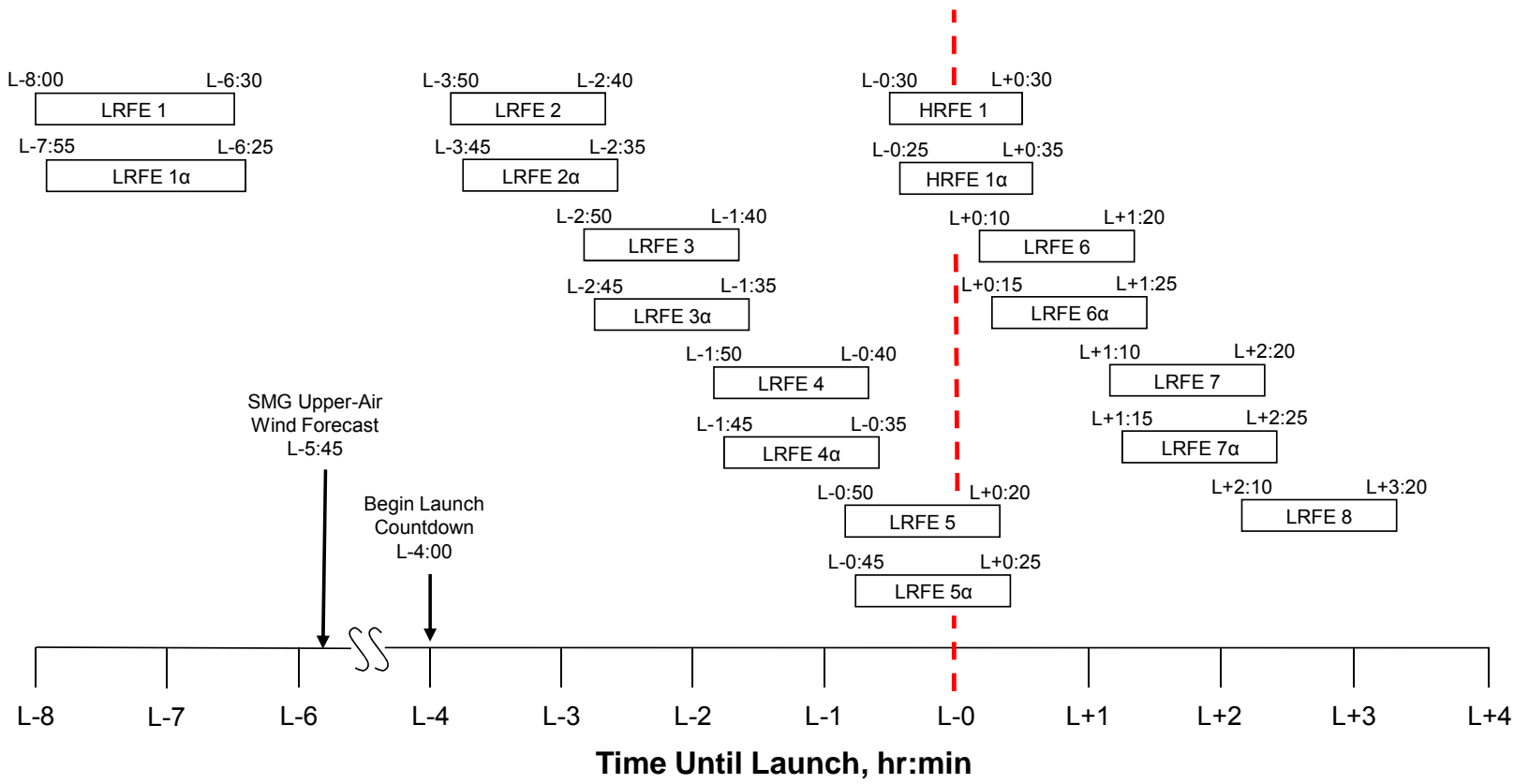

Figure 3. Balloon release schedule

\section{Wind and Atmospheric model}

The DOL trajectory simulation utilized wind and atmospheric models with data up to an altitude of $300 \mathrm{kft}$. Because the LRFE data did not extend to $300 \mathrm{kft}$, the models were created using a combination of LRFE data and data from the NASA MSFC Earth Global Reference Atmospheric Model - 2007, version 1.4 (GRAM 2007). LRFE data was transitioned into GRAM 2007 nominal October wind and thermodynamic data over $15 \mathrm{kft}$ of altitude change starting from the LRFE's highest data point. For the initial load assessment, the LRFE data extended up to the LRFE's maximum altitude of $100 \mathrm{kft}$. However, during the launch countdown, the LRFE data available for the assessment only extended to $65 \mathrm{kft}$.

The wind profile generated from the combination of LRFE and GRAM 2007 data was filtered to remove frequency content that would not persist for more than 3.5 hours $^{4}$. Wind features that did not persist were referred to as wind in-persistence. Filtering was done to be consistent with the development of STEL dispersions discussed in Section C-3. Once filtered, the wind and atmospheric models were generated in the DOL simulation format.

\section{B. DOL Trajectory Simulation}

The DOL trajectory simulation was a 6-DOF, rigid body simulation of a nominal FTV flying through the measured DOL winds and atmosphere. The simulation was developed in the Program to Optimize Simulated Trajectories II (POST II) ${ }^{5}$ by incorporating Ares I-X specific GNC, aerodynamic, propulsion, mass, atmospheric, 
and winds models into the POST trajectory simulation framework. The simulation's wind and atmospheric models were updated during each load assessment by linking to wind and atmospheric models developed from the LRFE. The only FTV system parameter not simulated at its monthly mean value was the RSRM propellant mean bulk temperature (PMBT). The RSRM PMBT was affected by temperatures in the Vehicle Assembly Building (VAB) during the FTV stack up and atmospheric conditions while at the pad. As a result, the PMBT was updated to an L-24 hour predicted value based on temperature measurements of the RSRM motor casing while in the VAB and at the pad.

The DOL trajectory simulation generated DOL parameters necessary for the STEL load calculation. The parameters included altitude, dynamic pressure, Mach number, total angle of attack, RSRM thrust components, and RSRM chamber pressure. The simulation was linked to the STEL analysis through an output data file. The trajectory parameters were used to calculate STEL loads at 0.1 second intervals throughout the ascent.

The effect of FTV system, wind, and atmospheric dispersions on the dynamic and STEL loads was determined pre-flight. The dynamic load dispersions were determined from Monte Carlo analyses that included system, wind, and atmospheric dispersions or from bounding cases. The dynamic loads Monte Carlo analyses wind and atmospheric dispersions were generated from the GRAM 2007 KSC Range Reference Atmosphere model and encompassed dispersions for the entire launch season. The effect of system dispersions on STEL load was determined with a Monte Carlo analysis that only included system dispersions. The effect of wind and atmospheric thermodynamic (density, pressure, temperature) dispersions was determined in separate analyses that used historical wind pairs for the fall launch season. The process used to determine the effect of wind and atmospheric dispersions on STEL loads is discussed in section C-3.

\section{Coupled Loads Analysis (CLA)}

The CLA consisted of performing individual aeroelastic and dynamic analyses and combining the loads from those analyses into a single set of forces and moments. The dynamic loads were calculated before flight over 10second time periods from 15 to 125 seconds (RSRM burnout). A 10 second interval was chosen because the FTV dynamic characteristics and dynamic loading did not change enough during that time period to affect the dynamic response. STEL loads were determined at 53 stations along the FTV at 0.1 second intervals throughout the ascent using inputs from the DOL trajectory simulation. The coupled loads were calculated as the combination of the STEL load with the maximum dynamic load that occurred during each 10 second period. The loads combination, STEL analysis and the dynamic analyses are described below.

\section{Loads Combination}

The forces and moments calculated from the DOL STEL analysis were combined with dynamic forces and moments using a loads combination equation. Dispersions in the STEL and dynamic loads were included in the load combination. The loads combination was a statistical combination of all loads that could occur simultaneously. There was no perfect way to perform loads combination. Some methods do a good job of matching flight data, yet are not mathematically rigorous. Other methods are mathematically rigorous while being conservative. As the Constellation Program did not establish a formal loads combination methodology, and since Ares I-X was a test flight, a conservative statistical approach that separates the random loads into a mean and dispersed value and combines them as random quantities was chosen ${ }^{6}$. Because the STEL load was not a random load and the random dynamic loads and dispersions were not a function of DOL conditions, the dynamic loads and dispersions could be combined into a single set of forces and moments before flight. Thus the loads combination used to determine DOL coupled loads was reduced to Eq. 1 where Dynamic + RSS(dispersions) represents the combination of all mean dynamic loads and all dispersions.

$$
\begin{aligned}
& \text { Equation } 1 \text { DOL Load Combination } \\
& \text { Coupled Load }=\text { STEL }_{D O L}+[\text { Dynamic }+R S S(\text { dispersion s })]_{\text {preflight }} \\
& \text { where Coupled Load }=\left[\begin{array}{c}
F_{X} \\
F_{Y} \\
F_{Z} \\
M_{X} \\
M_{Y} \\
M_{Z}
\end{array}\right]
\end{aligned}
$$




\section{CLA structural model}

The STEL and dynamic analyses were conducted using an integrated vehicle finite element model created by combining finite element models of the three main FTV subsystems: FS, USS, and Crew Module/Launch Abort System Simulator (CM/LAS). The model's structural coordinate frame was defined with its origin forward of the LAS tower, the $\mathrm{X}$ axis pointing rearward along the axial centerline of the FTV, the $\mathrm{Y}$ axis pointing opposite the system tunnels, and the $\mathrm{Z}$ axis completing the right hand rule. The FS model consisted of the FS aft skirt/nozzle section, four segments loaded with fuel, a fifth segment without fuel, a forward skirt, a forward skirt extension, and the frustum. The USS model consisted of the outer structure of the eight cylinders bolted together to simulate the Ares I US. The USS model included a representation of the ballast in the first and seventh cylinders necessary to obtain similarity to the mass properties of the Ares I operational vehicle. The CM/LAS model consisted of the structure representing the outer mold line of the Ares I CM/LAS. The integrated vehicle finite element model is shown in Fig. 4.

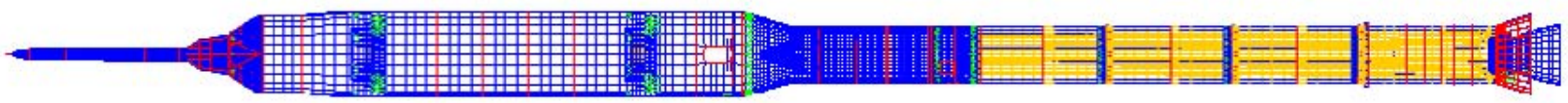

\section{Figure 4. FTV finite element model}

\section{STEL analysis}

Whenever the FTV flew at a non-zero angle of attack, the aerodynamic forces resulted in a destabilizing rigid body moment that was countered by the FTV's control system in order to maintain a prescribed attitude as a function of altitude. The bending moment induced by these two sets of forces deformed the FTV's shape which resulted in a variation in angle of attack along the FTV. An aeroelastic approach that accounted for the deformation's effect on aerodynamic loading was required for the calculation of forces and moments in the primary structure. STEL analysis is an aeroelastic analysis that incorporates the effects of vehicle deformation on local angle of attack. The DOL coupled loads analysis used a closed form STEL analysis rather than iterating to determine forces and moments resulting from aerodynamic loads. Since the angle of attack and the aerodynamic loads changed slowly relative to the FTV modal characteristics, the STEL analysis was treated as quasi-static.

The STEL analysis first calculated distributed aerodynamic and RSRM pressure loads using inputs passed from the DOL trajectory simulation. The inputs included rigid-body total angle of attack, dynamic pressure, Mach number, and RSRM chamber pressure. The RSRM chamber pressure was applied to the faces of the propellant or the exposed case in the FS structural model. The trajectory parameters were used in conjunction with local aerodynamic

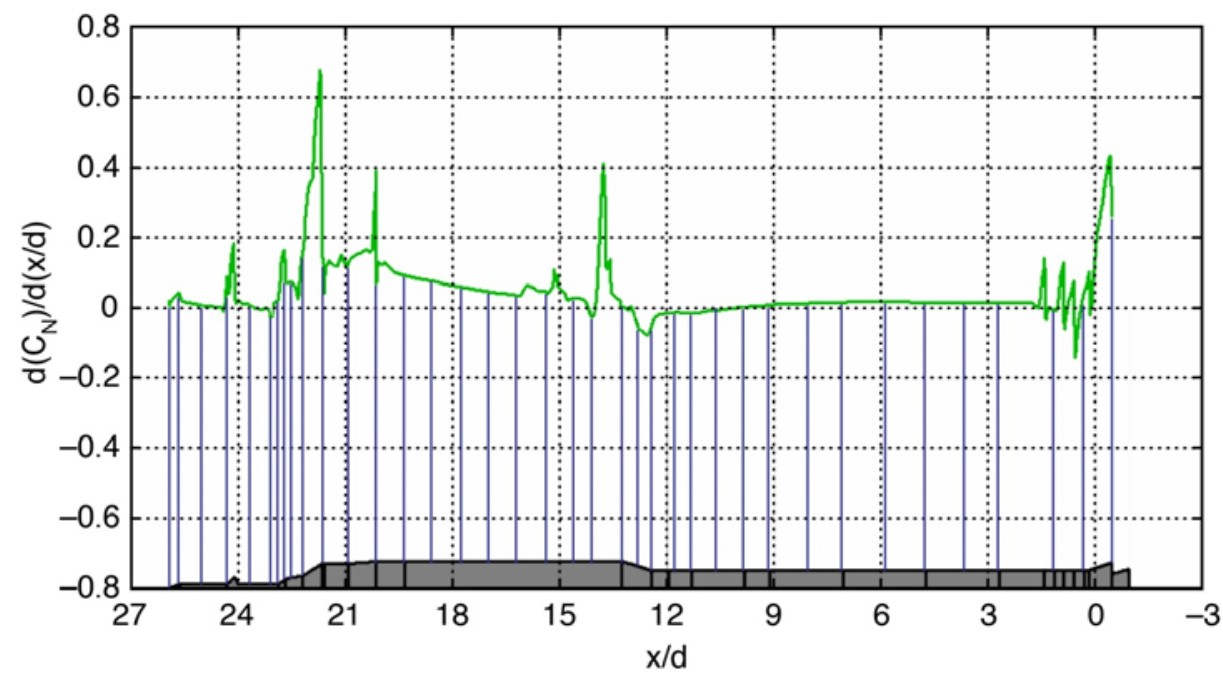

Figure 5. FTV distributed normal force coefficient

coefficients to calculate aerodynamic loading at 39 stations along the centerline of the vehicle. Total angle of attack was used in the analysis because of roll uncertainty during flight. The nodal coefficients were developed by integrating distributed aerodynamics between the station points. An example of the distributed normal force coefficient is shown in Fig. 5. The vertical blue lines between the FTV and distributed normal force coefficient curve represent the location of the station points. The slope of the vehicle aerodynamic coefficients with respect to angle of attack was linear which simplified the closed form STEL analysis.

The STEL analysis determined forces and moments in the FTV axial and lateral directions at 53 stations along the length of the FTV. The axial STEL loads were determined from the distributed axial aerodynamic forces and the 
RSRM pressure. The lateral STEL loads were determined from the distributed normal aerodynamic forces and the lateral thrust force at the gimbal required to trim the vehicle. Because of the roll uncertainty, the lateral STEL loads were determined in both the XY and XZ planes. The STEL loads were calculated at 0.1 second intervals over each 10 second time interval used in the dynamic analyses. The STEL analysis used trajectory parameters from the DOL simulation mentioned above in conjunction with pre-calculated structural matrices that represented the FTV characteristics during each interval to determine STEL loads.

The dispersion in STEL loads was determined from q-alpha total dispersions that result from FTV system parameter uncertainty, wind in-persistence, and the difference between the GRAM and DOL high altitude winds. A STEL analysis was conducted using the q-alpha total dispersion to determine the STEL dispersion from the October nominal trajectory STEL forces and moments. The method to determine the q-alpha total dispersion due to FTV system parameter uncertainty, wind in-persistence, and the difference between the GRAM and DOL high altitude winds is described below.

A Monte Carlo analysis was used to determine the q-alpha total dispersion due to FTV system uncertainty. The Monte Carlo analysis dispersed FTV system parameters but did not disperse wind or atmosphere. Instead, the analysis used nominal October wind and atmospheric conditions to isolate the effect of the system uncertainty on qalpha total. The 99.865 percentile q-alpha total dispersion from the nominal due to FTV system uncertainties is shown in Fig. 6 for 10 second intervals from 15 seconds to 125 seconds.
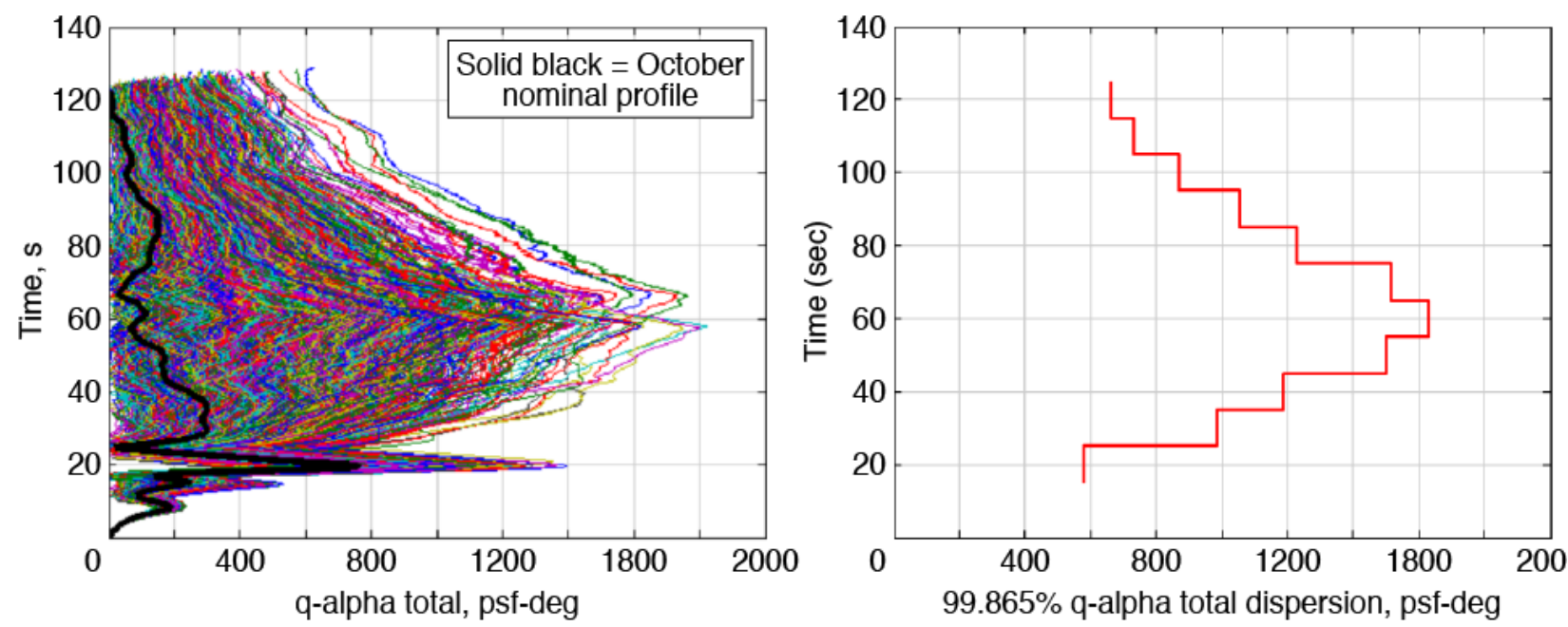

Figure 6. FTV system uncertainty q-alpha total dispersion

The q-alpha total dispersion due to wind in-persistence was determined using historical 3.5 hour wind pairs for the transition seasons (April to May and October to December) obtained from the SSP's Critical Math Model Database $^{7}$. This allowed the load assessment results to be valid for 3.5 hours after balloon release. The wind pairs consisted of two measured wind profiles whose measurements were taken 3.5 hours apart and provided a measure of wind in-persistence over that time. The wind in-persistence dispersion was quantified by determining the difference in q-alpha total resulting from flight through the winds in each pair. The first of the pair was filtered as described in section A to remove features that would not persist beyond 3.5 hours and give wind features that were common to both wind profiles. The second of the pair was unfiltered. The q-alpha total difference between the second of the pair relative to the first of the pair $\left(2^{\text {nd }}-1^{\text {st }}\right)$ represented the change in q-alpha total due to changes in the wind that could occur over 3.5 hours. A statistical analysis of the differences during each 10-second time interval was performed to determine the 99.865 percentile difference. The wind in-persistence q-alpha total dispersion was defined as the 99.865 percentile level of the q-alpha total differences. Figure 7 shows the q-alpha total for the first and second wind of each wind pair and the resulting 99.865 percentile dispersion for each time interval. 

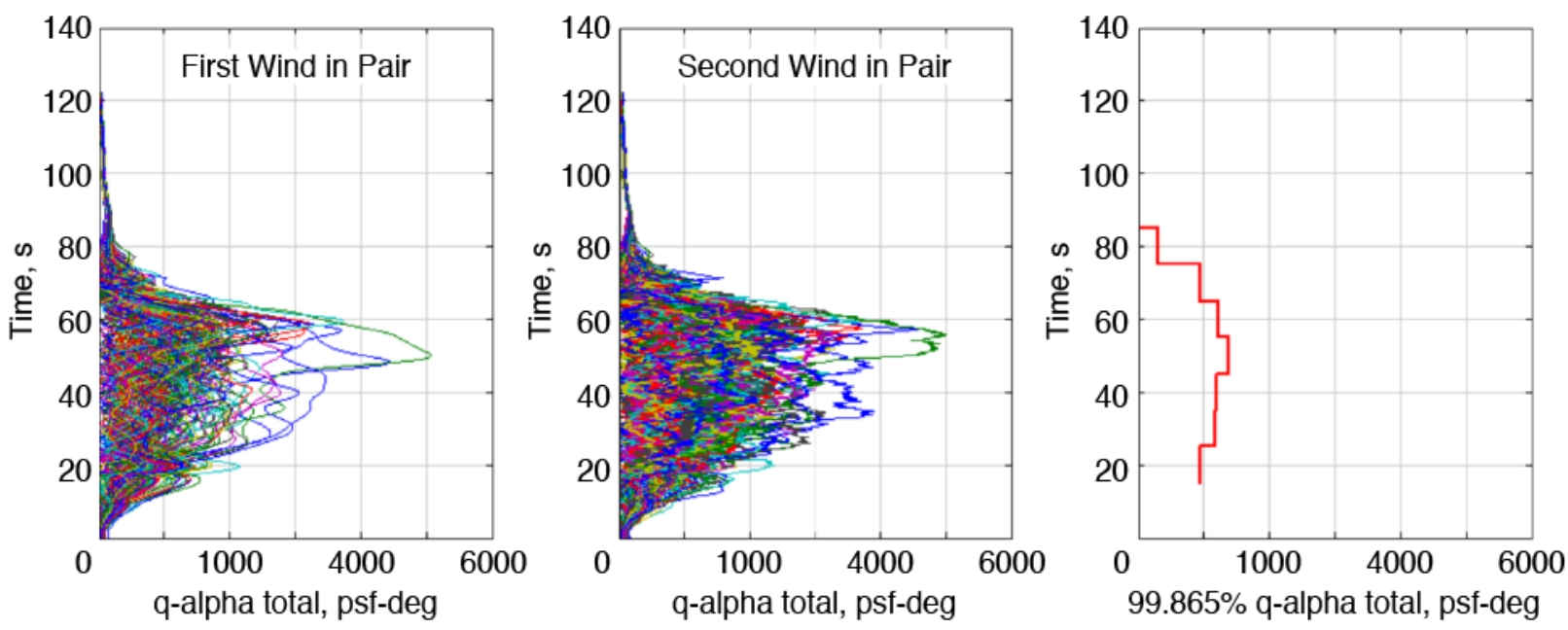

Figure 7. Wind in-persistence q-alpha total dispersion

The q-alpha total dispersion due to the difference between the GRAM and DOL high altitude winds was determined using the same basic process used for wind in-persistence described above. A historical database of high altitude winds was used but the database did not contain matched pairs measured at a known difference in time. Wind pairs were created by ramping each wind profile in the database into the GRAM nominal October wind as described in Section A. The high altitude wind ramped into the GRAM wind was used as the first in the pair and the same high altitude database wind with no modifications was used as the second in the pair. The first of the pair was filtered as described in section A. A statistical analysis of the q-alpha total differences between the second of the pair relative to the first of the pair $\left(2^{\text {nd }}-1^{\text {st }}\right)$ was performed to determine the 99.865 percentile level of the q-alpha total differences for each 10 -second time interval. The high altitude q-alpha total dispersion was defined as the 99.865 percentile level of the q-alpha total differences. The q-alpha total for the first and second wind of each wind pair and the resulting 99.865 percentile dispersion for each time interval are shown in Fig 8.
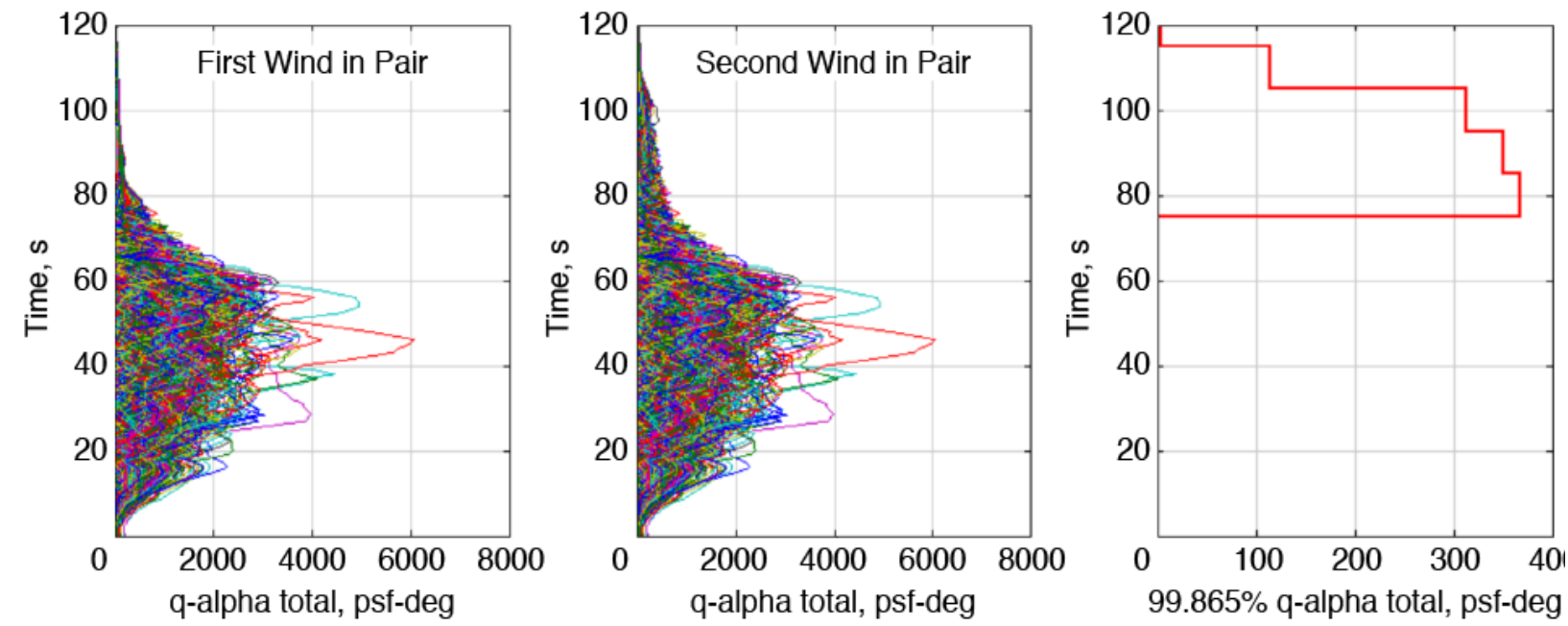

Figure 8. High altitude wind q-alpha total dispersion

The combined q-alpha total dispersion was calculated as the RSS of the FTV system, wind in-persistence, and high altitude wind dispersions and is shown in Fig. 9. The 99.865 q-alpha total dispersion was added to the October nominal q-alpha total and used in a STEL analysis to determine the corresponding STEL loads. The STEL load dispersion was defined as the difference from the October nominal trajectory STEL load. 


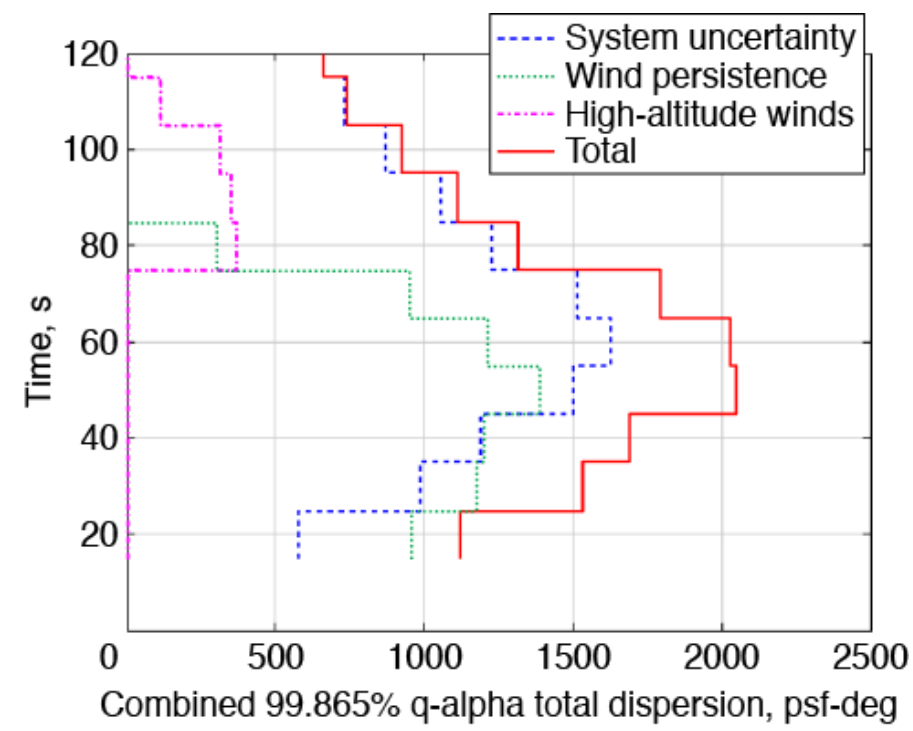

Figure 9. Combined q-alpha total dispersion

\section{Dynamic analyses}

Dynamic analyses were conducted pre-flight to determine the dynamic loads resulting from the FTV's response to wind gusts, aeroacoustic buffeting, RoCS firings, thrust vectoring during maneuvering, and RSRM internal acoustic modes. A dynamic analysis was performed for each dynamic load. The gust analysis determined loads resulting from transient wind features that have wavelengths long enough to tune with the FTV modes but are too short to be captured by STEL. The buffet analysis captured loads from the flow-induced fluctuating pressures. In particular, the FTV's diameter changes at the Crew Module (CM) and frustum produced flow separations that resulted in high buffet loads. A thrust oscillation (TO) analysis was performed to determine loads resulting from RSRM internal acoustic modes. During the RSRM burn, standing acoustical waves develop in the combustion chamber that produce chamber pressure oscillations and hence TO. TOs act as sinusoidal forcing functions in discrete frequency bands. An analysis was performed to determine the loads produced as a result of RoCS firings required to maintain the roll attitude. Last, an analysis was performed to determine the loads resulting from prescribed maneuvers such as the pitch-over maneuver executed to start down-range travel.

\section{Running Loads}

Tensile axial, compressive axial, and shear running loads were computed at the 53 stations along the FTV every 0.1 second as shown in Eq. 2 through Eq. 4. The maximum tensile axial, compressive axial, and shear running load that occurred during 10 second intervals from 15 seconds to 125 seconds defined the FTV's running load for that interval and was used to calculate a CR for each running load as a function of time and station as shown in Eq. 5 through Eq. 7. The allowable running loads were obtained from load envelopes published in the Ares I-X Structures Data Book and design calculations.

$$
\begin{aligned}
& \text { Equation } 2 \text { Tensile Axial Running Load } \\
& N_{X \max }=\max \left(\frac{F_{X}}{2 \pi r}+\frac{\sqrt{M_{Y}^{2}+M_{Z}^{2}}}{\pi r^{2}}\right)
\end{aligned}
$$

\section{Equation 3 Compressive Axial Running Load}

$$
N_{X \min }=\min \left(\frac{F_{X}}{2 \pi r}-\frac{\sqrt{M_{Y}^{2}+M_{Z}^{2}}}{\pi r^{2}}\right)
$$




$$
\begin{gathered}
\text { Equation } 4 \text { Shear Running Load } \\
N_{S}=\max \left(\frac{M_{X}}{2 \pi r^{2}}+\frac{2 \sqrt{F_{Y}^{2}+F_{Z}^{2}}}{\pi r}\right)
\end{gathered}
$$

$$
\begin{gathered}
\text { Equation } 5 \text { Maximum Running Load CR } \\
C R(t)=D O L N_{X \max }(t) / \text { Allowable } N_{X \max }(t)
\end{gathered}
$$

$$
\begin{gathered}
\text { Equation } 6 \text { Minimum Running Load CR } \\
C R(t)=D O L N_{X \min }(t) / \text { Allowable } N_{X \min }(t)
\end{gathered}
$$

Equation 7 Shear Running Load CR

$$
C R(t)=D O L N_{S \max }(t) / \text { Allowable } N_{S \max }(t)
$$

\section{E. Loads Assessment Summary}

Matlab and Perl scripts were used to automate the development of winds and atmospheric models, running the DOL trajectory simulation, performing the CLA, and generating the trajectory and loads reports. The LRFE data, winds and atmospheric models, trajectory simulation, and CLA were linked through input/output data files. The automated process allowed load assessments to be completed within approximately 15 minutes of receiving the LRFE data. The trajectory report consisted of wind and FTV flight parameters data and time history plots while the loads report consisted of running loads and CR data and plots. A go/no-go report for the weather launch director was developed within approximately 15 minutes of completing the loads assessment.

The results of the load assessments were verified by an Ascent Dynamics Team at Johnson Space Center (JSC). The JSC Ascent Dynamics Team independently performed each analysis conducted in the loads assessment.

Selected plots from one of the October 28, 2009 trajectory and loads reports are shown in the following figures. The North and East wind components are shown in Fig. 10 with the North wind component shown on the left and the East wind component shown on the right. The plot compares the LRFE winds and filtered winds to the October nominal wind. Note that the filtered winds shown in red blends into the GRAM wind at $80 \mathrm{kft}$ and lies on top of the GRAM wind shown in green. The dynamic pressure and q-alpha total resulting from the filtered wind are shown in Fig. 11 and Fig. 12. The effect of the DOL wind on dynamic pressure and q-alpha total can be seen in those figures. In Fig. 12, the red curve represents the q-alpha total obtained from adding the 99.865 percentile q-alpha total dispersion to the DOL q-alpha total. 

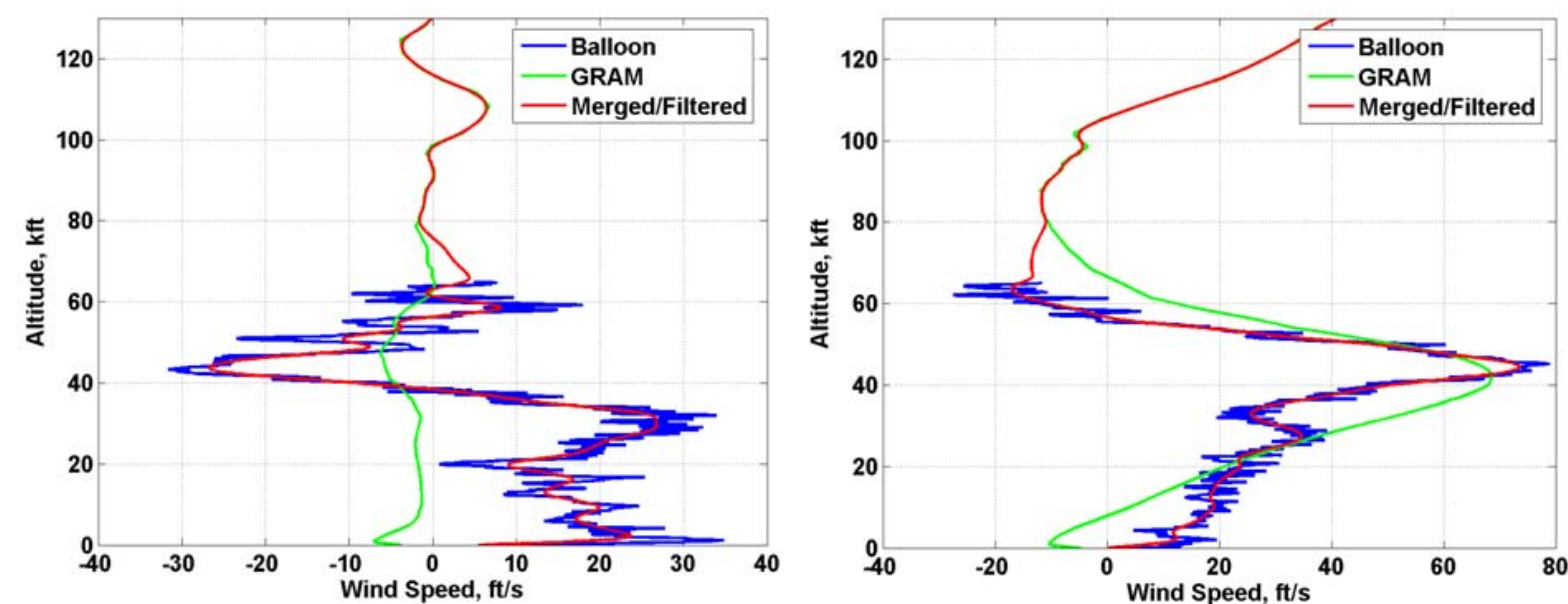

Figure 10. DOL North and East wind components
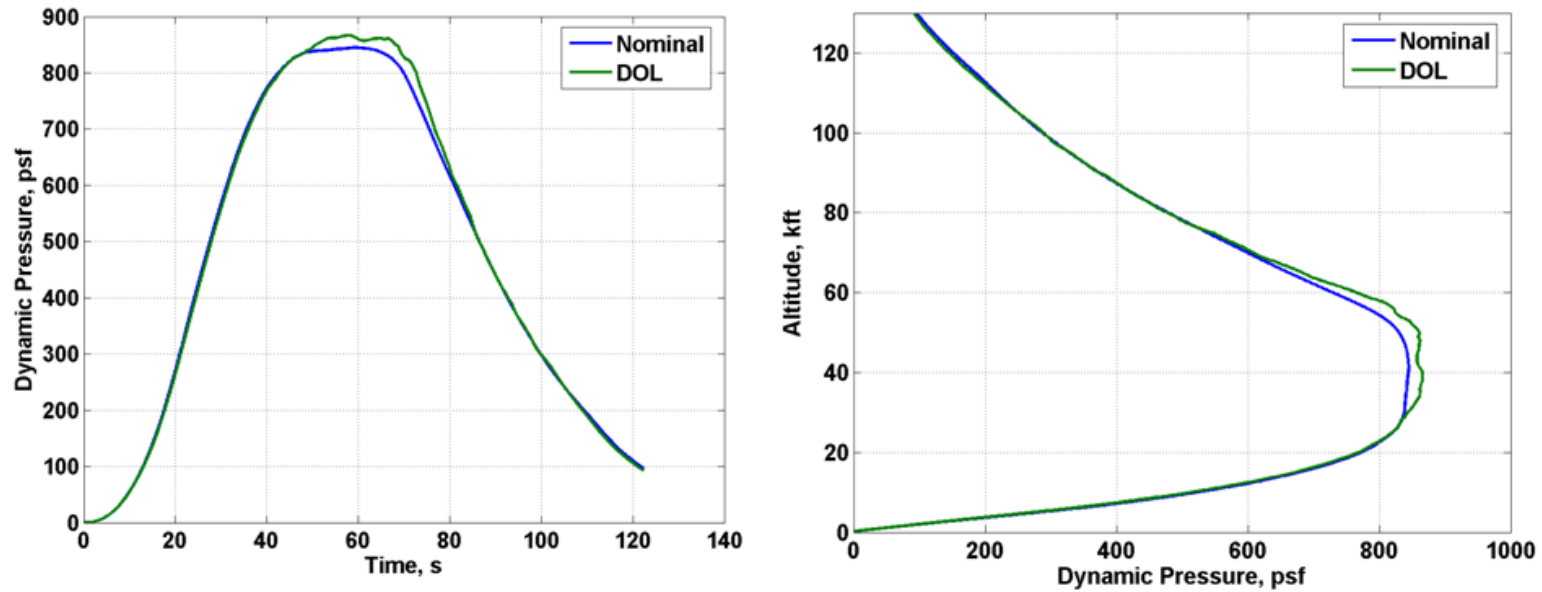

Figure 11. DOL dynamic pressure
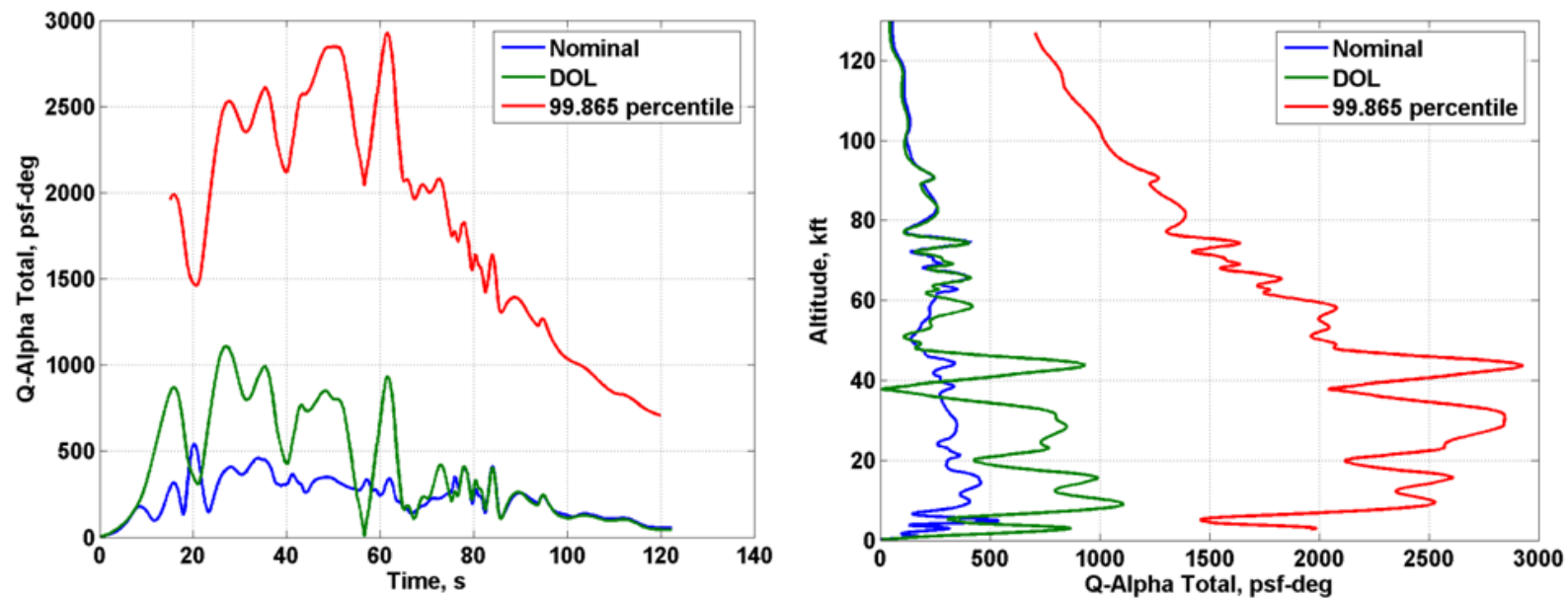

Figure 12. DOL q-alpha total

12

American Institute of Aeronautics and Astronautics 
The running loads along the length of the FTV are shown in Fig. 13. The running loads are compared to the FTV tensile, compressive, and shear strength limits shown as dashed lines. The large tensile load and small compressive load from station number 2600 to 4000 is due to the effect of the pressure in the FS combustion chamber. Note that the step change in tensile and compressive loads is the result of chamber pressure effects ending at the end of the combustion chamber. The compressive and shear running loads exceed the FTV strength at some station locations below 1000 inches. The plot on the right shows a magnification of that region.
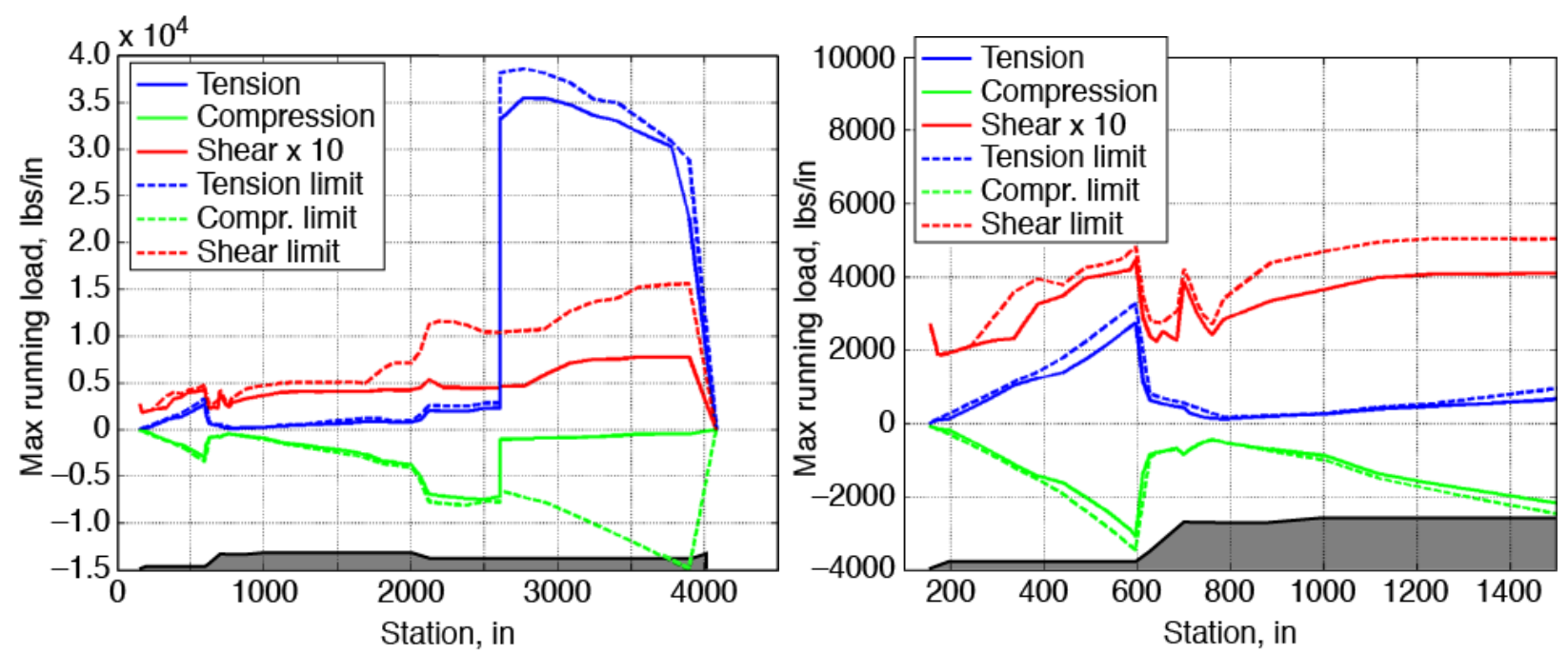

\section{Figure 13. DOL running load}

The CRs corresponding to the running loads are shown in Fig. 14. CR as a function of station reports the maximum CR that occurred at each station throughout the ascent. The $\mathrm{CR}$ as a function of time shows the largest CR that occurred at any station at each time point throughout the ascent. The CR plots show that the compressive and shear running loads exceeded their limits around 40 seconds (approximately $20 \mathrm{kft}$ ). The compressive running load exceedance was approximately 5\% and occurred at stations around 750 inches and 200 inches. The shear running load exceedance was approximately $3 \%$ and occurred at stations around 250 inches.
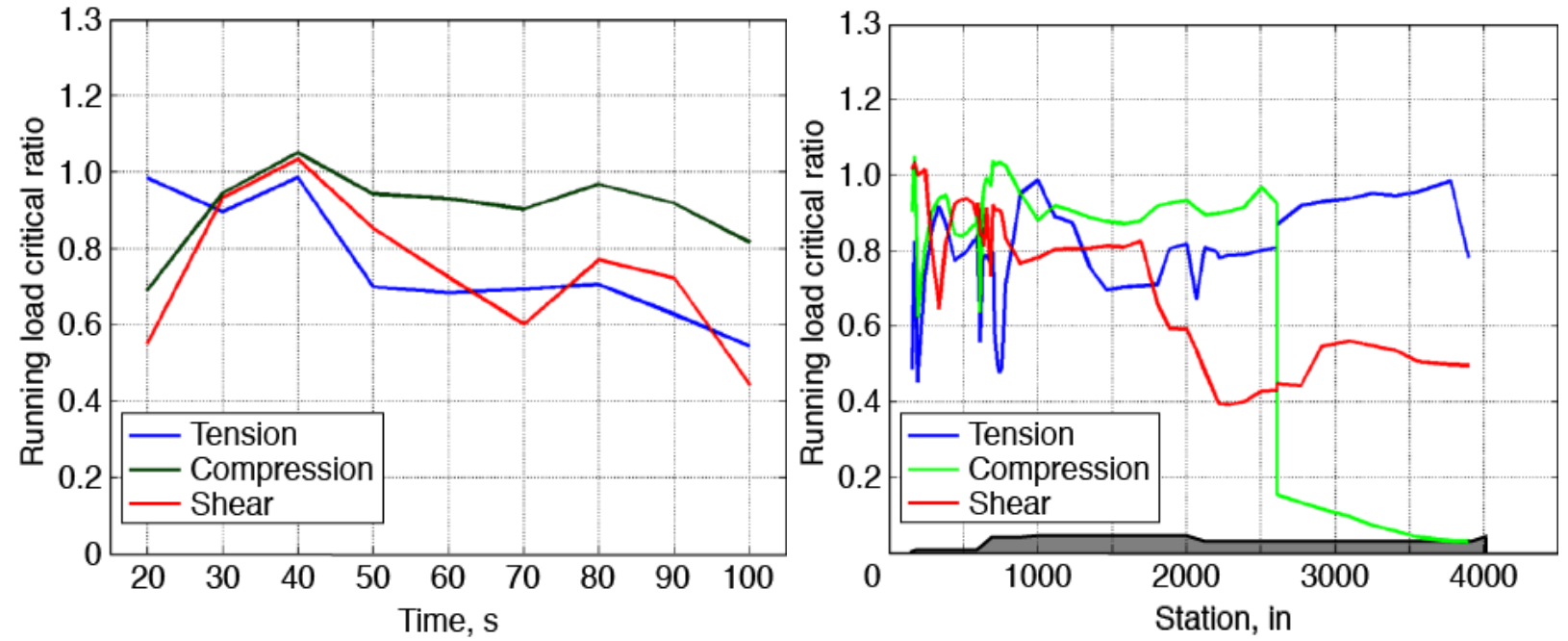

Figure 14. DOL CR

The CR exceeded the launch commit criteria and resulted in a trajectory no-go call for launch. Because the exceedance was small, $5 \%$ or less, a CR waiver was recommended. The CR waiver rationale was developed specifying the running load components that exceeded their limit, the magnitude of exceedances, the location of exceedances, the time of the exceedances, and the duration of the exceedances. The IPTs responsible for certifying the structure's ability to withstand launch loads reviewed the waiver and determined the structural load limits had sufficient margin to allow the exceedances. 
Expressing the flight loads in terms of the structure's running load limits proved to be essential to launching during the first launch opportunity and reducing launch costs. The FTV's first launch opportunity lasted only two days due to other previously scheduled launches. The first day was no-go for launch due to violation of a triboelectrification launch commit criteria and the second day's winds produced a no-go call. The CLA provided the necessary details about the exceedance to allow the IPTs to grant a CR waiver in a timely manner and proceed with launch. If a waiver could not have been processed in time, the launch would have been delayed into December resulting in roll back cost and the cost of maintaining the launch team.

\section{Conclusion}

A DOL flight loads assessment technique was successfully developed for the Ares I-X test flight that determined coupled loads from the combination of dynamic and quasi-static aeroelastic loads and expressed them in terms of running load in the primary structure. The loads assessment could be completed in approximately 15 minutes and fit within the time constraints of the Ares I-X launch count schedule. The technique's approach of expressing loads in terms of running load components rather than a q-alpha indicator allowed an exceedance to be accurately quantified as a percentage of the allowable limit and allowed the identification of the specific running load components that exceeded their limit. That information proved beneficial in development of a waiver to the loads launch commit criteria that was readily usable by the IPTs and allowed them too clear Ares I-X for launch during its initial two day launch period.

\section{Acknowledgments}

The author extends thanks to Frank Leahy of MSFC and Paul Duffin of JSC for their help with the paper. The author also wishes to thank the MSFC Natural Environments Team members Barry Roberts, Frank Leahy, Stan Adelfang, Kevin McGrath, Robert Barbre, Ryan Decker, and Gary Dempse and the JSC Ascent Dynamics Team members Henry Cordova, Paul Duffin, Dan Pupperi, Carl Merry, Mike Harvey, Tom Schmidt, and Mike Scott for their contributions to Ares I-X DOL loads assessment technique and support of this paper. The teams provided invaluable guidance in regard to DOL processes and support in the development of loads assessment process. The teams were essential to achieving a successful Ares I-X DOL flight loads assessment.

\section{References}

${ }^{1}$ Starr, B.R., Gumbert, C.R., and Tartabini, P.V. “Ares I-X Test Flight Reference Trajectory Development," JANNAF Joint Propulsion Meeting, Arlington VA, April 2011.

${ }^{2}$ Roberts, B.C., McGrath, K.M., "Upper Atmospheric Monitoring for Ares I-X Ascent Loads and Trajectory Evaluation on the Day-of-Launch", 2009-3781, $1^{\text {st }}$ AIAA Atmospheric and Space Environments Conference, San Antonio, TX, June 22-25, 2009.

3 Justus, C.G. and Leslie, F.W., "The NASA MSFC Earth Global Reference Atmospheric Model - 2007 Version", NASA/TM-2008-215581, November 2008.

${ }^{4}$ Cordova, H.S., Leahy, F.B., Barbre, R.E., "Use of Smoothed Measured Winds to Predict and Assess Day of Launch Environments", submitted for publication, AIAA Atmospheric Flight Mechanics Conference, Portland, OR, August 8-11, 2011.

${ }^{5}$ Striepe, S.A., Powell, R.W., Desai, P.N., Queen, E.M. "Program to Optimize Simulated Trajectories (POST II) Utilization Manual”, Vol. II, Version 1.1.6G, Denver, CO. January 2004.

${ }^{6}$ Sako, B.H., Kabe, A.M., and Lee, S.S., "Statistical Combination of Time-Varying Loads", AIAA Journal, Vol. 47, No. 10, October 2009, pages 2338-2349.

${ }^{7}$ Wind Pairs Database, Shuttle Critical Math Models Database, CMM ID 00261, [online, cited July 2011] http://sspapps.unitedspacealliance.com/cmm_pprd/SilverStream/Pages/pgCMMReport18RomanT.html?id=4170 